

\section{Concepciones geográficas que sostienen los profesores en las aulas de las escuelas secundarias públicas de la ciudad de Paraná, Entre Ríos}

\section{Teacher's Geographic Conceptions from Paraná City, Entre Rios Public High Schools}

Concepções geográficas sustentadas pelos professores nas salas de aula das escolas secundárias públicas da cidade de Paraná, Entre Rios

Alejandra María Teresita Marek*

Alejandra Anahí Laiker

Jacqueline Daiana Dalinger

\section{Resumen}

El interés puesto en el trabajo de investigación "Concepciones geográficas que sustentan los docentes de geografía de profesores de escuelas secundarias de Paraná, Entre Ríos, Argentina", que se presentará en el presente texto, se debe a la necesidad de dar continuidad a una investigación anterior denominada "¿Cuáles son los discursos geográficos de los docentes en las aulas de octavo año de la EGB?", estudio de caso de tres escuelas de Paraná. La presente investigación pone el acento en los profesores egresa- dos entre 2005-2008 de la carrera del Profesorado en Geografía de la Facultad de Humanidades, Artes y Ciencias Sociales (FHAyCS) dependiente de la Universidad Autónoma de Entre Ríos (Uader).

\section{Palabras clave}

concepciones geográficas; geografía escolar; enseñanza del territorio.

* Profesoras de la Universidad de Entre Ríos, Argentina. 


\section{Abstract}

The interest put in the qualified work of investigation " geographical Conceptions that there sustain the teachers of teachers' Geography of secondary schools of Parana, Between Rivers Argentina ", that will appear in the present text it is necessary to the need to give continuity in a previous investigation called which are the geographical speeches of the teachers in the classrooms of eighth year of the EGB? Study of case of three schools of the depart-

\section{Resumo}

O interesse colocado no trabalho de pesquisa "Concepções geográficas sustentadas pelos professores nas salas de aula das escolas secundárias públicas da cidade de Paraná, Entre Rios, Argentina", apresentada no texto a seguir, surge da necessidade de continuar uma pesquisa anterior denominada "Quais são os discursos geográficos dos professores nas salas de aula de oitavo ano da EGB?", estudo de caso de três escolas de Paraná. A presente ment Parana. The present investigation puts the accent in the teachers gone away between the years 2005-2008 of the career of the Professorship in Geography of the Faculty of Humanities, Arts and Social Sciences (FHAyCS) dependent on Autonomous University of Between Rivers (Uader).

\section{Keywords}

geographic conceptions: school geography; territory teaching pesquisa está enfocada nos professores formados entre 2005 e 2008 na Licenciatura em Geografia pela Faculdade de Humanidades, Artes e Ciências Sociais (FHAyCS) da Universidade Autônoma de Entre Rios (Uader).

\section{Palavras-chave}

concepções geográficas; geografía escolar; ensino do território 


\section{Introducción}

Es necesario subrayar que en nuestra carrera del Profesorado, la geografía está planteada como una ciencia social cuyo objeto de estudio se centra en la enseñanza de las diferentes problemáticas socioterritoriales.

Esta investigación pretende ser exploratoria. Se buscará información a través del análisis de las concepciones epistemológicas registradas en las prácticas áulicas de los docentes de las escuelas secundarias, en sus planificaciones anuales, en entrevistas a los docentes, entre otras fuentes de información.

Consideramos que nuestro trabajo será relevante y significativo, por un lado, si logramos hallar indicios que nos permitan avanzar en los procesos de cambios que experimenta la enseñanza de la geografía escolar entrerriana, y, por otro, si reflexionamos sobre nuestras propias prácticas docentes universitarias.

Es importante aclarar que el diseño de este proyecto de investigación fue presentado en el III Coloquio Internacional de Investigadores en Didáctica de la Geografía- LADGEO desarrollado en la Universidad de Buenos Aires en el 2014. Finalmente, se considera fundamental hacer circular la producción de conocimiento entre los colegas latinoamericanos. Por ello este trabajo se presentó también en el III Encuentro Iberoamericano de Investigación en Didáctica de las Ciencias Sociales del 5, 6 y 7 de octubre del 2016 en Santiago de Chile.

\section{Aproximaciones al problema de investigación}

En esta ponencia presentamos algunos avances del proyecto de investigación y desarrollo de inserción (Pidin) denominado "Concepciones geográficas que sostienen los profesores en las aulas de las escuelas secundarias públicas de la ciudad de Paraná, Entre Ríos". En esta dirección, es necesario destacar que el presente proceso de investigación se realizó a partir del trabajo de campo, en el cual se realizaron las siguientes actividades destinadas específicamente a la obtención de información:

- Ensayos, aplicaciones y evaluaciones de las técnicas e instrumentos diseñados para la recolección de la información.

- Realización de los registros y observaciones de clases, llegando a una totalidad de 51 registros.

- Elaboración de entrevistas abiertas y semiestructuradas a los profesores encargados de los cursos en los cuales se realizaron los registros y observaciones de clase.

- Análisis de las planificaciones áulicas.
- Análisis, interpretación e integración de los resultados a partir de la recolección, el grabado, la transcripción, la lectura, la codificación (abierta, axial y selectiva) y la elaboración de memos.

De esta manera, se avanzó con la construcción de las categorías y subcategorías de análisis, actividad que pudo realizarse a partir de la interpretación y análisis de los datos obtenidos (tabla 1).

Tabla 1. Relación categorías y subcategorías para el análisis

\begin{tabular}{|c|c|}
\hline Categoría & Subcategorías \\
\hline $\begin{array}{l}\text { Concepciones } \\
\text { geográficas }\end{array}$ & - Objeto de estudio \\
\hline $\begin{array}{c}\text { Objetivo de la geografía } \\
\text { escolar }\end{array}$ & Formación espacial de niños, niñas y jóvenes. \\
\hline $\begin{array}{c}\text { Contenidos geográficos } \\
\text { escolares }\end{array}$ & $\begin{array}{l}\text { - Selección de contenidos geográficos } \\
\text { escolares } \\
\text { - Organización de los contenidos escolares } \\
\text { - Preferencia por la enseñanza de } \\
\text { determinados contenidos geográficos } \\
\text { escolares }\end{array}$ \\
\hline $\begin{array}{l}\text { Metodología de la } \\
\text { enseñanza escolar }\end{array}$ & $\begin{array}{l}\text { - Transposición del saber geográfico en } \\
\text { situaciones didácticas } \\
\text { - Recursos y estrategias didácticas }\end{array}$ \\
\hline
\end{tabular}

A partir de las presentes categorías de análisis, el equipo de investigación se encuentra en aquella instancia de trabajo que refiere a la interpretación y la integración de los resultados, con la finalidad de triangular la información recabada y aproximarnos a interpretar las tendencias de las concepciones geográficas que sostienen los profesores de geografía en el aula.

En este sentido, es necesario especificar que para realizar el análisis de los datos se segmentaron los grupos de muestra según las diferentes instituciones públicas de la ciudad de Paraná, Entre Ríos, denominándolas "profesora A, B y C". Por lo tanto, las aproximaciones que el equipo realizó hasta el presente periodo (junio, 2016) refieren a:

- Predominancia de contenidos geográficos vinculados a los saberes académicos sustentados en diferentes concepciones epistemológicas de la geografía.

- Las prácticas docentes y la articulación entre los recursos didácticos y la enseñanza de la geografía.

- Clases con diferentes estructuras, ya que algunas de ellas presentan una estructura cerrada, otras más flexibles, con presencia de enseñanza de contenidos conceptuales transmitidos con reiteración de recursos didácticos.

- La construcción conceptual de las temáticas se lleva adelante apelando directamente a mencionar el concepto sin indagar en los 
saberes previos y en otras situaciones. Se presenta una repetición de rescate de ideas previas, con moderada relación posterior al concepto a desarrollar.

En relación con el contenido específicamente conceptual, esas ideas no se vuelven a retomar para su construcción, sino que se realiza una reproducción de este.

- Los autores de los textos trabajados en clase son reconocidos intelectuales de la ciencia geográfica. En este sentido, se puede observar que las docentes utilizan enunciados ya aceptados por la academia.

- Se observan escasos desafíos cognitivos en las estrategias: las actividades o consignas de trabajo suelen ser repetitivas, y en enunciados generales y orales o escritos en la pizarra (se reitera el dictado y el uso de videos). Así mismo, se pudo analizar en ocasiones la transcripción de consignas a la carpeta desde los libros y manuales en circulación.

- Se analiza y advierte secuenciaciones didácticas pensadas en relación con sencillas estrategias utilizadas en clase según la capacidad psicológica de los alumnos.

- Escasa problematización del conocimiento con abundancia de ejemplos para trabajar la temática.

- Las dinámicas de las clases se manifiestan visiblemente en realizar correctamente la actividad planteada: leer y releer, para poder reproducir tal cual está en el libro de texto.

- Existe un predominio de actividades vinculadas con información de datos y hechos puntuales por encima de aquellas relacionadas con la cooperación, el intercambio, la conceptualización y la explicación.

A partir del análisis de las categorías construidas en relación con los diferentes registros de observación y entrevistas, si bien falta el análisis de otros instrumentos, se podría considerar en principio que en la enseñanza de la geografía propuesta por los profesores seleccionados para esta investigación, hay una coexistencia de enfoques epistemológicos en la disciplina, presentándose algunos desde el positivismo, determinismo y neopositivismo. Además, en cuanto a la incorporación de lenguajes que se utilizan, si bien son diversos - presencia del lenguaje verbal, visual, escrito, cartográfico-, la utilización que se realiza sobre ellos está condicionada por los contenidos conceptuales que se presentan. Por lo tanto, "cada opción teórica acarrea determinadas alternativas de acción desde el punto de vista pedagógico-didáctico" (Fernández, 2007, p. 19).

\section{Fundamentación teórica}

Consideramos que uno de los rasgos característicos de la geografía en la carrera del Profesorado, correspondiente a la Facultad de Humanidades, Artes y Ciencias Sociales de la Uader, es que esta se concibe como una ciencia social cuyo objeto de estudio se centra en la enseñanza de los diferentes problemas socioterritoriales. Sin embargo, coincidimos en que la ciencia geográfica se encuentra inscripta dentro del carácter pluri/multiparadigmático al que esencialmente pertenecen el conjunto de las ciencias sociales, de lo cual se desprende una cuestión para tener en cuenta: la variabilidad teórica -en su interdependencia con sus diferentes paradigmas - que caracteriza a la ciencias sociales. Livingstone afirma que

[...] el término Geografía ha significado y significa hoy diferentes cosas para diferentes personas. Para algunos, evoca imágenes de lugares distantes y exploradores intrépidos yendo a donde nadie había ido antes. Para otros, el geógrafo es considerado una persona que posee un conocimiento de carácter enciclopédico referido a los ríos más largos, una suerte de atlas hablante de gran valor para los programas de preguntas y respuestas de la televisión y no mucho más. Finalmente, existen otros para quienes la geografía es la disciplina que trabaja con diagramas y globos: se dice que, si la historia se ocupa de los hombres, la geografía se ocupa de los mapas. En realidad, es muy probable que hoy en día los geógrafos profesionales rechacen todas estas nociones del sentido común como definiciones de la propia disciplina y proporcionarán una explicación propia respecto a lo que estudia la geografía. (Livingstone, 1992, p. 1).

Cordero y Svarzman sostienen que "la presencia de diversas concepciones que estructuran la enseñanza de la Geografía, remiten a los fundamentos epistemológicos que, de manera implícita o explícita, están presentes en las prácticas docentes" (Cordero y Svarzman, 2007, p. 121).

A partir de lo expuesto en líneas anteriores, principalmente en lo que respecta al carácter pluri/multiparadigmático que ostenta la geografía, consideramos importante para la presente investigación analizar las concepciones epistemológicas que sostienen los docentes de geografía en sus prácticas docentes en las escuelas secundarias públicas de Paraná.

En relación con lo planteado, Moraes (1987) es quien sostiene que es en la concepción filosófica y metodológica del positivismo donde encuentran su fundamento todas las corrientes de la geografía tradicional. Los postulados del positivismo van a ser el basamento sobre el cual se erige el pensamiento geográfico tradicional, dándole unidad:

Una primera manifestación de esta filiación está en la reducción de la realidad al mundo de los sentidos, esto es, en circunscribir todo trabajo científico al dominio de la apariencia de los fenómenos. De ahí la limitación de todos los procedimientos de análisis a la inducción, señalada como el único camino para la explicación científica. La descripción, la enumeración y clasificación de los hechos referentes al espacio son momentos de su apreciación, pero la Geografía Tradicional se limitó a ellos, como si ellos cumpliesen toda la tarea del trabajo científico. Esta concepción, presente en todas las definiciones expuestas, frustró la posibilidad a la Geografía de llegar a un conocimiento 
más generalizador, que no quedase a cuestas del formalismo topológico. En síntesis, que traspasase la descripción y clasificación de fenómenos. (Moraes, 1987, p. 18).

Al contrario de la geografía tradicional, Moraes (1987) afirma que el movimiento de renovación no posee una unidad. El mosaico de la geografía renovada es bastante diversificado, abarcando un abanico muy amplio de concepciones.

Entretanto, es posible agruparlas, en función de sus propósitos y de sus posicionamientos políticos, en dos grandes conjuntos: uno puede ser denominado geografía pragmática, otro geografía crítica. Es por el compromiso social contenido en ellas, por el proyecto histórico que vinculan, en fin, por la ideología que alimentan y a los intereses concretos a los que sirven, que se torna posible agruparlas. Común a cada una se encuentran

Propuestas singulares y procedimientos distintos. En las dos vertientes, aparecen posturas filosóficas, luego fundamentos metodológicos, diversificados. La unidad ético-política no implica directamente perspectivas unitarias, con respecto a métodos. Entretanto, esto no invalida la característica común de cada conjunto, que es transparente, por ejemplo, en los horizontes de crítica a la Geografía tradicional. (Moraes, 1987, p. 85).

La geografía pragmática ataca principalmente el carácter no práctico de la geografía tradicional. Los autores pragmáticos proponen una visión prospectiva, volcada hacia el futuro, que instrumentalice una geografía aplicada:

De esta manera, su intención general es de una renovación metodológica, o de búsqueda de nuevas técnicas y un nuevo lenguaje, que dé cuenta de las nuevas tareas planteadas por el planeamiento. La finalidad explícita es crear una tecnología geográfica, un móvil utilitario. (Moraes, 1987, p. 89).

La otra concepción, dentro de la geografía renovada, es la geografía crítica. La designación de crítica nos habla al respecto de una postura frente a la realidad, frente al orden constituido. Los autores que se encuentran dentro de esta concepción proponen una geografía militante, que luche por una sociedad más justa. Moraes afirma que

[...] son los que piensan el análisis geográfico como un instrumento de liberación del hombre. En cuanto al nivel académico, los geógrafos que se ubican dentro de esta concepción, critican el empirismo exacerbado de la Geografía Tradicional, que sigue manteniendo el análisis en el mundo de las apariencias, así como la búsqueda de un objeto autónomo, la idea absoluta de ley, la no diferenciación de las distintas cualidades de los fenómenos humanos, etc. (1987, p. 101).

A través de las diferentes concepciones expuestas, consideramos que será necesario destacar que
La escuela lejos de ser canónica y repetitiva, es una activa constructora de las disciplinas escolares, ya que las pautas para la enseñanza no le llegan linealmente desde fuera. Dentro de la escuela, en los tiempos de la escuela, a partir de la interacción entre alumnos y profesores, con aportes de los programas o diseños y el de propuestas que van circulando, se toman decisiones y se definen contenidos. Y eso que se construye dentro de la escuela es lo que Chervel llama disciplina escolar. (Villa, 2009, p. 3).

En este sentido, focalizándonos en el caso específico de la geografía, el autor François Audigier utiliza esta idea para analizar la geografía escolar como una construcción de la escuela y no como una transformación o simplificación del saber sabio que ayuda a ponerlo al alcance de niños y jóvenes. La disciplina escolar de geografía, como todas las disciplinas escolares, resulta estrechamente ligada a las finalidades de las instituciones educativas, ya que son estas las que definen qué enseñar en la escuela y por qué la geografía se enseña en ella (Audigier, 1992).

Por lo tanto, consideramos significativo avanzar teóricamente en la práctica docente. Achilli afirma que esta es

[...] el trabajo que el docente desarrolla cotidianamente en determinadas y concretas condiciones sociales, históricas e institucionales, adquiriendo una significación tanto para la sociedad como para el propio docente. Trabajo que si bien está definido por la práctica pedagógica, va mucho más allá de ella al involucrar una compleja red de actividades y relaciones que la traspasa. En tanto, la práctica pedagógica es como el proceso que se desarrolla en el contexto del aula en el que se pone de manifiesto una determinada relación maestro-alumno, centrada en el enseñar y el aprender. (Achilli, 1986, p. 6).

De esta manera, nuestro análisis se centrará en las concepciones geográficas que sostienen los profesores egresados durante la cohorte 20052008 de la carrera Profesorado en Geografía de la Universidad Autónoma de Entre Ríos en las aulas de las escuelas secundarias públicas de la ciudad de Paraná.

Para ello, se partirá del buceo bibliográfico a fin de recoger obras teóricas, investigaciones y publicaciones, que se analizarán en forma crítica y responsable, con lo que se desea identificar aquel material bibliográfico de tipo relevante y pertinente vinculado a nuestro objetivo de análisis.

En la selección de investigaciones científicas, que se vinculan con nuestra problemática, han sido escasas las que utilizan el término concepción geográfica. Sin embargo, aparecen otras investigaciones con términos vinculados a tradiciones geográficas, discursos geográficos, perspectivas geográficas, entre otros.

Por tanto, se trabajará en la presente investigación con concepciones geográficas, entendiéndolas como una categoría fundamental que se 
basa en los fundamentos epistemológicos de la ciencia geográfica, permitiéndonos interpretar la incidencia de las teorías geográficas que subyacen en la enseñanza de la geografía escolar llevadas a cabo por los profesores egresados durante la cohorte 2005-2008 de la carrera Profesorado en Geografía de la Universidad Autónoma de Entre Ríos en las aulas de las escuelas secundarias públicas de la ciudad de Paraná.

\section{Objetivos}

\section{Generales}

- Conocer y analizar las concepciones geográficas que sostienen los docentes de geografía en sus prácticas pedagógicas de las escuelas secundarias públicas de Paraná.

- Elaborar tipos o mapas conceptuales con los resultados obtenidos.

\section{Específicos}

- Establecer relaciones respecto a las concepciones geográficas que sostienen los docentes entre las entrevistas, las planificaciones anuales y las observaciones áulicas.

- Identificar las tendencias teóricas en las prácticas áulicas de geografía a partir de las fuentes de información seleccionadas.

- Describir las concepciones geográficas presentes en las prácticas áulicas de los docentes egresados de la FHAyCS.

\section{Metodología}

Consideradas las características del objeto a investigar y su correlación con los objetivos planteados, se llevará a cabo un estudio profundo y exhaustivo que permita obtener un conocimiento amplio y detallado sobre las concepciones geográficas que están presentes en las aulas de las escuelas públicas secundarias de Paraná. Por todo ello es que esta investigación será de tipo exploratorio y descriptivo de corte cualitativo.

Para Mason (1996, p. 4), toda investigación cualitativa no puede ser reducida a un conjunto simple y prescriptivo de principios; por tal motivo, señala que toda investigación que se desarrolle bajo este encuadre está atravesada por una posición filosófica que es ampliamente interpretativa en el sentido que se interesa en las formas en las que el mundo social es interpretado, comprendido, experimentado y producido; basada en métodos de generación de datos flexibles y sensibles al contexto social en el que se producen; $y$, por último y no menos importante, sostenida por métodos de análisis y explicación que abarcan la comprensión de la complejidad, el detalle y el contexto. Así, con el enfoque cualitativo se busca alcanzar la solidez que yace en el conocimiento que proporcionan los docentes de geografía en sus aulas, para ir desenvolviendo los significados que los participantes expondrán en sus clases.

Sánchez Crespo afirma que por población (universo de estudio) comúnmente se entiende "un conjunto de unidades, para las cuales se desea obtener información" (Sánchez, 1971, p. 11). Las unidades pueden ser personas, familias, viviendas, escuelas, organizaciones, artículos de prensa, y toda la información vendrá dictada por los objetivos de la investigación. Por tal motivo, la población de nuestro estudio está constituida por los profesores egresados durante la cohorte 2005-2008 de la carrera Profesorado en Geografía de la Universidad Autónoma de Entre Ríos.

La selección de esta obedece al siguiente motivo: todos los egresados que formarán parte de la muestra realizaron la carrera según el plan de estudio del 2003.

La definición de una unidad de análisis implica la ubicación en un espacio y tiempo concreto con el fin de lograr una contextualización; en este proyecto se tomarán aquellos docentes que estén realizando sus prácticas pedagógicas en las escuelas secundarias públicas en Paraná y que hayan egresado durante la cohorte 2005-2008 de la carrera Profesorado en Geografía. Según la naturaleza de nuestra investigación (exploratoria-descriptiva), de corte cualitativo, se trabajará con un reducido número de unidades de análisis. De esta manera, se privilegia más la validez del conocimiento obtenido, que la posibilidad de generalizar características medibles de una muestra probabilística a todo el universo.

En virtud de lo expuesto, las unidades a incluir en nuestra muestra constituyen una decisión en la que participan diversos factores. Nos apoyamos en Cea D’Ancona (2001), quien establece criterios que conducen a decidir sobre el diseño de la muestra, como el tiempo y los recursos disponibles, la modalidad de muestra seleccionada, la diversidad de los análisis de datos prevista, el nivel de confianza de la estimación muestral; y en función a la clase de interrogante y a los objetivos que persigue el siguiente trabajo, el tipo de muestra corresponde al no probabilístico, estratégico o de conveniencia, ya que se seguirán criterios establecidos por el equipo de investigación, diferentes de la aleatorización. Este tipo de muestreo, según Cea D’Ancona (2001), es el más apropiado para las indagaciones exploratorias y de estudios cualitativos, debido a su interés en profundizar la información aportada, en lugar de buscar la representatividad estadística.

El procedimiento de la selección muestral concluye cuando se llega a la saturación teórica, esto es, cuando la información comienza a ser redundante, no aportando ningún nuevo punto de vista analítico, por lo que se decide terminar la recogida de información. 
Para concluir en la saturación teórica dentro de las características de la investigación, el trabajo de campo constituye una parte central dentro de dicho proceso.

Para poder analizar las concepciones geográficas de los docentes es necesario estar en contacto con ellos, en su lugar de trabajo; esto implica descripción, análisis, interpretación y conceptualización, actividades que marcarán el inicio del trabajo de campo.

Cada una de las técnicas y fuentes mencionadas brindarán informaciones y datos que, a través de su análisis exhaustivo, permitirá la creación de categorías analíticas para luego ser comparadas y cruzadas teóricamente, y de esta forma generar conclusiones que posibilitarán la descripción e interpretación de nuestro objeto de estudio.

\section{Fases de la investigación}

La presente investigación se desenvolverá a través de varias fases o etapas:

1. Primera fase: para dar cumplimiento a esta fase de investigación se procederá a elegir la documentación que dará soporte a la inves- tigación y que conformará el marco conceptual de las categorías principales, las subcategorías y las dimensiones.

2. Segunda fase: hace referencia a la investigación en campo dividida en tres líneas de análisis: documentos (revisión y análisis de los programas, las planificaciones anuales, la carpeta de los alumnos), observación y registros de prácticas áulicas en las clases de geografía y entrevistas, y cuestionarios autoadministrados a los diversos actores que integran la comunidad educativa que en forma directa o indirecta se relacionan con nuestra unidad de análisis. Se llevará un registro de las categorías que permanecen iguales y cuales han sufrido modificaciones y de qué tipo; todo esto se volcará en un diario de campo.

3. Tercera fase: se refiere al análisis, interpretación e integración de los resultados. Esta fase se llevará a cabo a través del desarrollo de conexiones entre las categorías y subcategorías existentes y las que surgirán mediante el soporte del Atlas.ti.

\section{Referencias}

Achilli, E. (1986). La práctica docente: una interpretación desde los saberes del maestro. Cuadernos de Formación Docente. Argentina: Universidad Nacional De Rosario.

Audigier, F. (1992). Pensar la geografía escolar. Un reto para la didáctica. Documentos De Análisis Geográfico.

Cea D’Ancona, M. A. (2001). Metodología cuantitativa. Estrategias y técnicas de investigación social. Madrid: Síntesis.

Cordero, S. y Svarzman, J. (2007). Hacer geografía en la escuela. reflexiones y aportes para el trabajo en el aula. Buenos Aires: Novedades Educativas.

Fernández Caso, M. V. (2007). Geografía, nuevos temas nuevas preguntas: un temario para su enseñanza. Buenos Aires: Biblos.

Livingstone, D. (1992). Una breve historia de la geografía. En P. Zusman Unc (trad.), The Student's companion to geography. Londres: Blackwell.

Mason, J. (1996). Qualitative researching. Londres: Sage.

Moraes, A. C. R. (1987). Geografía. Pequeña historia crítica. San Pablo: Hucitec.

Sánchez Crespo, J. L. (1971). Principios elementales de muestreo y estimación de proporciones. Madrid: INE.

Villa, A. (2009). La construcción didáctica como forma y parte de los contenidos a enseñar. Ponencia presentada en XI Jornadas de Investigación del Centro de Investigaciones y del Departamento de Geografía, 12 y 13 de noviembre. La Plata, Argentina. 